Published in final edited form as:

Nat Med. 2018 December ; 24(12): 1845-1851. doi:10.1038/s41591-018-0232-2.

\title{
Radiotherapy induces responses of lung cancer to CTLA-4 blockade
}

\author{
Silvia C. Formenti ${ }^{1}{ }^{,}$, Nils-Petter Rudqvist ${ }^{\# 1}$, Encouse Golden ${ }^{\# 1,14}$, Benjamin Cooper ${ }^{2}$, Erik \\ Wennerberg $^{1}$, Claire Lhuillier ${ }^{1}$, Claire Vanpouille-Box ${ }^{1}$, Kent Friedman ${ }^{3}$, Lucas Ferrari de \\ Andrade $^{4,5}$, Kai W. Wucherpfennig ${ }^{4,5}$, Adriana Heguy ${ }^{6,7}$, Naoko Imai ${ }^{8}$, Sacha Gnjatic ${ }^{8}$, Ryan \\ O. Emerson ${ }^{9}$, Xi Kathy Zhou ${ }^{10}$, Tuo Zhang ${ }^{11}$, Abraham Chachoua ${ }^{12}$, and Sandra \\ Demaria $^{1,13, *}$ \\ ${ }^{1}$ Department of Radiation Oncology, Weill Cornell Medicine, New York, New York, USA. \\ ${ }^{2}$ Department of Radiation Oncology, New York University School of Medicine, New York, New \\ York, USA. \\ ${ }^{3}$ Department of Radiology, New York University School of Medicine, New York, New York, USA \\ ${ }^{4}$ Department of Cancer Immunology and Virology, Dana-Farber Cancer Institute, Boston, \\ Massachusetts, USA \\ ${ }^{5}$ Department of Microbiology and Immunobiology, Harvard Medical School, Boston, \\ Massachusetts, USA \\ ${ }^{6}$ Department of Pathology, New York University School of Medicine, New York, New York, USA. \\ ${ }^{7}$ Genome Technology Center, Division of Advanced research Technologies, NYU Langone Health, \\ New York, New York, USA. \\ ${ }^{8}$ Tisch Cancer Institute, Hematology/Oncology, Immunology, Icahn School of Medicine at Mount \\ Sinai, New York, NY, USA. \\ ${ }^{9}$ Adaptive Biotechnologies, Seattle, Washington, USA \\ ${ }^{10}$ Division of Biostatistics and Epidemiology, Department of Healthcare Policy and Research, \\ Weill Cornell Medicine, New York, New York, USA
}

\footnotetext{
Users may view, print, copy, and download text and data-mine the content in such documents, for the purposes of academic research, subject always to the full Conditions of use: http://www.nature.com/authors/editorial_policies/license.html\#terms

*Correspondence to: szd3005@med.cornell.edu; formenti@med.cornell.edu.

Author contributions: S.C.F., A.C. and S.D. conceived and designed the clinical protocol. S.C.F. and S.D. designed the correlative studies. E.G., B.C. and K.F. contributed to patients enrollment and treatment and/or response evaluation. N.-P.R. developed the neoantigen prediction pipeline and analyzed the TCR repertoire. A.H. and T.Z. helped with WES and RNAseq. E.W., C.L., N.I. and S.G. performed flow cytometry and functional T cell studies. C.V.-B. contributed to cytokine measurements and the patient-derived tumor xenograft experiment. L.F.d.A. and K.W.W. evaluated sMICA and antibodies. R.O.E. helped with TCR repertoire analysis.

X.K.Z. performed the statistical evaluations. S.D., S.C.F. and N.-P.R. wrote the manuscript. All authors had final responsibility for the decision to submit this report as written for publication.

Competing interests: Bristol Meyer Squibb did not have any role in the design, data collection and analysis, interpretation of results and preparation of the manuscript. Potential conflict of interest: Full-time employment and equity ownership at Adaptive Biotechnologies Corporation (R.O.E.). Prior honorarium for consulting from Neon Therapeutics and research funding from Agenus (S.G.). Prior honorarium for consulting from AstraZeneca, AbbVie, Lytix Biopharma, Cytune. Research grants from Nanobiotix, and Lytix Biophrama (S.D.). Prior honorarium for consulting/speaker from AstraZeneca, Merck, Regeneron, Bayer, Serono, and research funding from Varian, Merck, Bristol Meyer Squibb (S.C.F.).
} 
${ }^{11}$ Department of Microbiology and Immunology, Weill Cornell Medicine, New York, New York, USA

${ }^{12}$ Department of Medicine, New York University School of Medicine, New York, New York, USA

${ }^{13}$ Department of Pathology and Laboratory Medicine, Weill Cornell Medicine, New York, New York, USA

${ }^{14}$ Present address: Department of Radiation Oncology, University of California, San Francisco, CA 94143, USA.

\# These authors contributed equally to this work.

\section{Introductory paragraph}

Focal radiation therapy (RT) enhances systemic responses to anti-CTLA-4 antibodies in preclinical studies and in some melanoma patients ${ }^{1-3}$, but its efficacy in inducing systemic responses (abscopal responses) against tumors unresponsive to CTLA-4 blockade remained uncertain. RT promotes the activation of anti-tumor T cells, an effect dependent on type I interferon induction in the irradiated tumor ${ }^{4-6}$. The latter is essential for achieving abscopal responses in murine cancers ${ }^{6}$. The mechanisms underlying abscopal responses in patients treated with RT and CTLA-4 blockade remain unclear. Here we report that RT and CTLA-4 blockade induced systemic anti-tumor $\mathrm{T}$ cells in chemo-refractory metastatic non-small cell lung cancer (NSCLC), where anti-CTLA-4 antibodies had failed to demonstrate significant efficacy alone or in combination with chemotherapy ${ }^{7,8}$. Objective responses were observed in $18 \%$ of enrolled patients, and $31 \%$ had disease control. Increased serum interferon- $\beta$ after radiation and early dynamic changes of blood $\mathrm{T}$ cell clones were the strongest response predictors, confirming pre-clinical mechanistic data. Functional analysis in one responding patient showed the rapid in vivo expansion of CD8 T cells recognizing a neoantigen encoded in a gene upregulated by radiation, supporting the hypothesis that one explanation for the abscopal response is radiation-induced exposure of immunogenic mutations to the immune system.

The choice of NSCLC for testing the combination of RT with the anti-CTLA-4 antibody, ipilimumab, was supported by a case of a complete and durable abscopal response to this combination, in a patient with metastatic NSCLC ${ }^{9}$. To prospectively evaluate RT to one metastasis (palliative dose, 6GyX5 or 9GyX3) and concurrent ipilimumab thirty-nine patients were enrolled between June 2014 and April 2015 (NCT02221739, Supplementary Table 1 and Fig. 1a). All patients had progressed after $\geq 1$ prior systemic treatment, and $41 \%$ had pre-existing brain metastases controlled by surgery or radiotherapy at study entry. One patient had received prior immunotherapy.

Twenty-one of 39 patients (54\%) completed 4 cycles of ipilimumab and could be evaluated at day 88 by Response Criteria In Solid Tumors (RECIST). Adverse events were consistent with ipilimumab-induced side effects, and the addition of RT did not modify them (Supplementary Table 2). One additional patient received four cycles but did not undergo response evaluation. Seventeen patients received less than 4 cycles because they either died $(n=8)$ or progressed $(n=9)$ before day 88 and were taken off treatment. Patients who did not complete treatment had a more advanced disease at study entry, with significantly more 
organs involved by metastasis, more frequently had bone metastases and had received more courses of prior chemotherapy (Supplementary Table 1).

Objective radiographic responses occurred in $18 \%$ of enrolled patients ( 7 of 39 patients) or $33 \%$ of evaluable patients ( 7 of 21 patients) with 2 complete (CR) and 5 partial (PR) responses (Fig. $1 \mathrm{~b}$ and $\mathrm{c}$ and Supplementary Tables 3 and 4). In addition, 5 patients had stable disease (SD) at evaluation. Thus, disease control (PR+CR+SD) was achieved in 12/39 (31\%) patients. At median follow up of 43 months for survivors (range: 38-47 months), the median overall survival (OS) for the entire cohort of 39 patients was 7.4 months (95\% CI: 4.4-12.6) (Fig. 1d and e). In patients who completed treatment the median OS was 13.0 months (95\% CI: 10.6-25.2) versus 3.0 months for those who did not (95\% CI: 2.5-3.5) (log-rank test $\mathrm{p}<0.001$ ) (Supplementary Fig. 1). In patients who achieved disease control median OS was 20.4 months (95\% CI: 12.9-not reached) compared to 3.5 months in patients who did not (95\% CI: 3.1-7.4) (log-rank test p<0.001) (Fig. 1f and g). Four patients who completed treatment (among which 3 achieved disease control) were alive at the time of last follow-up 38, 42, 44 and 47 months since study entry.

To investigate the mechanisms underlying an abscopal response to RT and ipilimumab tumor tissue and peripheral blood were analyzed. PD-L1 expression in pre-treatment tumor was not associated with response, as it was detected in 6/10 non-responders, including 3 patients with progressive disease at evaluation (PD) and 3 non-evaluable patients (NE) who did not complete treatment, and in 4/5 patients with disease control out of the 15 patients with tissue available for testing. Likewise, CD8 T cell infiltration was not associated with response (Supplementary Table 5 and Supplementary Fig. 2).

Baseline clinical parameters, including the neutrophil to lymphocyte ratio, previously shown to be higher in patients without abscopal response to RT and granulocyte-macrophage colony-stimulating factor ${ }^{10}$, were comparable in patients with or without disease control after completing treatment (Supplementary Fig. 3a). However, the proportion of patients with EGFR mutated cancers, a feature associated with low total mutation burden ${ }^{11}$ and poor response to anti-PD-1/PDL- ${ }^{12}$ was significantly higher in patients with PD compared to patients with disease control (Supplementary Table 6). Neither the radiation regimen used (9GyX3 versus 6GyX5) nor the location of the irradiated lesion was significantly associated with treatment response.

A panel of circulating soluble markers and immune cells (Supplementary Dataset 1) was analyzed in the 21 patients who completed treatment and were evaluable for response. At baseline, absolute lymphocyte count (ALC) was lower and regulatory $\mathrm{T}$ cells $\left(\mathrm{T}_{\text {REG }}\right)$ were higher in responding compared to SD and PD patients. ICOS expression was also higher in responders in both regulatory and conventional CD4 T cells (Supplementary Fig. 3b and Dataset 2). Changes in circulating soluble markers and immune cells occurring during treatment were evaluated by comparing values obtained at day 22 versus baseline. We also calculated the largest change from baseline in either direction (increase or decrease) in every parameter over the entire course of treatment, to account for different kinetics of change among patients (Supplementary Dataset 2). During treatment, most patients showed an increase in ICOS expression on CD4 T cells and in proliferation of CD8 and CD4 T cells, 
evidenced by Ki67 expression, irrespective of response. Thus, two known pharmacodynamic biomarkers for anti-CTLA-4 activity ${ }^{13,14}$ did not distinguish responders from nonresponders in this study. Expansion in $\mathrm{ICOS}^{+} \mathrm{T}_{\mathrm{REG}}$ in melanoma patients treated with interleukin- 2 was associated with worse outcome ${ }^{15}$ but in our patients this subset was similarly expanded in responders and non-responders, suggesting a possible role in mitigating ipilimumab-induced inflammation rather than anti-tumor responses. In contrast to a reported melanoma patient ${ }^{3}$, no significant changes in myeloid-derived suppressor cells were detected after RT in NSCLC patients. The percentage of PD- $1^{+}$CD8 T cells was increased during treatment in both responders and non-responders, whereas only responders showed a significant increase of PD $-1^{+} \mathrm{CD} 4 \mathrm{~T}$ cells. Approximately $20 \%$ of PD- $1^{+} \mathrm{T}$ cells were $\mathrm{Ki}^{+} 7^{+}$in both responders and non-responders, suggesting an activated rather than exhausted state (Supplementary Table 7). T-effector memory cells were increased in the CD8 and CD4 compartments, irrespective of response (Supplementary Fig. 4 and Dataset 2). CTLA-4 was expressed mainly on $\mathrm{T}_{\text {REG }}$ without significant changes during treatment (Supplementary Dataset 3).

Serum levels of galectin1, an immune suppressive tumor-secreted factor reported to be increased by radiation in NSCLC ${ }^{16}$, were comparable at baseline between responders and non-responders and did not change significantly at day 22 (Supplementary Fig. 3c).

Elevated serum levels of soluble major histocompatibility complex class I chain-related protein A (sMICA) at baseline were associated with resistance to CTLA-4 blockade in melanoma patients ${ }^{17}$. In our patients baseline levels of sMICA did not differ significantly between responders and non-responders. During treatment, sMICA levels increased significantly in patients with PD while responding patients showed an increase in antibodies against sMICA and sMICB (Supplementary Dataset 2 and Supplementary Fig. 3d). These antibodies were shown to block sMICA/B-mediated downregulation of NKG2D receptor on effector CD8 T and natural killer (NK) cells, and restore tumor response to ipilimumab in some patients ${ }^{18}$. An increase in NKG2D receptor expression was seen across all NK subsets only in patients with SD (Supplementary Dataset 2), and thus it could not be attributed to increased anti-sMICA/B antibodies seen only in patients with CR/PR. Anti-MICA/B antibodies could have different functions, including blocking MICA/B shedding from tumor cell surface ${ }^{19}$. RT-induced upregulation of surface MICA/B was shown to improve cancer cell killing by NK and CD8 T cells ${ }^{20,21}$, but no post-treatment tumor tissue was available to investigate this hypothesis.

Concordant with studies in mice $^{6}$, we found that RT induction of interferon- $\beta$ (IFN $\beta$ ) correlates with the abscopal response in this patient population. Serum IFN $\beta$ showed the most significant increase from baseline shortly after completion of RT (day 22) in the 7 responders (Fig. 2a, and Supplementary Dataset 2). IFN $\beta$ was also significantly but more modestly increased in the 5 patients with SD, whereas no significant increase occurred in the remaining 23 patients with PD and serum available for measurement, including those who did not complete treatment with ipilimumab.

Next, we evaluated $\mathrm{T}$ cell clonal representation in peripheral blood with deep sequencing of $\mathrm{T}$ cell receptor (TCR) CDR3 regions (TCR-seq). A significantly larger number of $\mathrm{T}$ cell 
clones were expanded at day 22 compared to baseline in responders than in patients with SD and PD (Fig. 2b). Responders also showed a significantly larger number of contracted clones (Fig. 2c). In a random Forest (RF) analysis the levels of IFN $\beta$ at day 22 and the TCR repertoire changes showed the highest predictive value (compared to other variables that were significantly altered at day 22) in classifying correctly the patients by response status: 7/7 responders, 6/8 patients with PD and 2/5 patients with SD were correctly assigned, and no patient with PD was classified as responder (Fig. 2d).

We next assessed the tumor specificity of the $\mathrm{T}$ cell clones that were expanded in the blood early after RT completion. For this, we performed TCR-seq on archival tumors from four patients representative of different response status: 1 CR (subject \#4), 1 with SD (\#32), and 2 with PD (\#36 and \#38). TCR sequences obtained from tumors were designated as TILTCRs and compared to the sequences in blood. In each of the four patients there were TILTCRs whose frequency increased from baseline over the course of treatment, but their number was markedly larger in the patient with CR (\#4) compared to the others (Fig. 3a). Twenty of the clones present in patient \#4 tumor were undetectable in the blood at baseline but showed a rapid increase during treatment and most remained elevated at follow-up. Patient \#32, who had SD, showed expansion of a smaller number of clones already present at baseline that remained elevated at follow-up. In marked contrast, in the blood of patients \#36 and 38, who had PD, there was an expansion of few pre-existing clones, and their increase was minimal and/or not sustained.

We next asked if the number of TIL-TCRs expanded by treatment in blood was proportional to the number of pre-existing tumor-infiltrated CD8 T cells. The highest density of CD8 T cells was found in patient \#36, who also showed the highest TCR clonality, while patient \#32 had very few CD8 T cells, and patients \#4 and \#38 had an intermediate number (Fig. $3 b)$. Hence, the quantity and quality of the pre-treatment $\mathrm{T}$ cell infiltrate was not related to the treatment-induced expansion of TIL-TCRs in peripheral blood (Figure 3b).

We then examined the TCR CDR3 amino acid sequences relatedness ${ }^{22,23}$ of the clones present in the two pre-treatment brain metastases of patient \#4. Out of 51 TIL-TCRs increased in blood (Fig. 3a) 19 were common to both metastases and were found in multiple branches of the dendrogram, consistent with the expansion of a diverse repertoire. The largest TIL-TCR that was increased in blood had distinct features as it was found in a separate branch in each tumor (Fig. 3c). Thus, the combination of RT and CTLA-4 blockade expanded a selected and diverse set of TCR clones.

In melanoma patients treated with ipilimumab and in NSCLC patients treated with antiPD-1 antibodies the mutational landscape is an independent determinant of response ${ }^{24-26}$. Thus, we assessed the number of antigenic mutations in the tumors of the 4 patients with different response to RT and ipilimumab. The number of single nucleotide missense variants (SNV) in patients \#4, 32, 36 and 38 ranged from 199 to 345, and frameshift mutations from 11 to 22 (Supplementary Fig. 5). The lowest number of predicted mutation-associated neoantigens was found in patients \#4 (CR) and \#38 (PD). There were no significant differences between the four patients in the MHC-I binding affinity, variant allele frequency, or the wild type/mutant ratio of peptide MHC-I binding affinity of the predicted neoepitopes. 
Expression levels of the genes encoding the predicted neoepitopes, based on the lung adenocarcinoma TCGA data, did not differ among the 4 patients. None of the tumors had mutated $T A P$ or $B 2 M$ genes (Supplementary Dataset 4), which are required for antigen presentation and are often mutated in immunotherapy-resistant tumors ${ }^{27}$. However, loss of heterozygosity at HLA-A and -C loci was found in patient \#4 (Supplementary Table 8). Finally, no somatic mutations were identified in major genes of the interferon- $\gamma$ pathway (JAK1/2, STAT1, IFNG, IFNGR1/2) in any of the 4 patients (Supplementary Dataset 4). Thus, known mechanisms of primary resistance to immune checkpoint inhibitors could not explain the lack of response in patients \#36 and \#38.

Although we did not find the global tumor mutation rates to correlate with response, we speculated that new tumor-specific $\mathrm{T}$ cell clones might be detected in patients who responded best to the combination of RT and anti-CTLA-4. To test this notion, we first tested the post-treatment peripheral blood of patient \#4 for reactivity against the 33 top candidate neoepitopes identified using a neoantigen prediction pipeline (Fig. 4a). Eight candidates were selected upon initial screening and two peptides (p15 and p16) were confirmed to be recognized by the patient's CD8 T cells by ELISpot assay (Fig. 4 b-d). Both peptides contain the same mutation but p15 was predicted to bind to HLA-A2402, while p16 was predicted to bind to HLA-C1203, the two alleles expressed by the tumor at the HLA-A and -C loci (Supplementary Table 8). While the mutation did not alter the predicted binding affinity of the peptides to the respective HLA alleles, the wild-type peptides did not elicit a response in ELISpot assay, indicating T cell specificity for the mutated peptides (Fig. 4d). We next interrogated the in vitro expanded T cells against these two peptides by TCR-seq. One clone from p15-reactive CD8 T cells and one clone from p16-reactive CD8 T cells were identified in the blood of patient \#4 (Fig. 4e and Supplementary Table 9). The clone reactive towards p16 was present in both pre-treatment metastases and its frequency in the blood went from barely detectable at baseline to markedly expand at day 22 remaining elevated at day 43 and at subsequent follow up more than 6 months later (Fig. 4e).

We were intrigued by the robust CD8 $\mathrm{T}$ cell response in patient \#4 and probed the biology of the protein from which the antigen was derived. The mutation recognized by the expanded clones derives from the protein, karyopherin a2 (KPNA2, also known as importin-a). Previous work has shown that KPNA2 is upregulated by RT in human colorectal cancer cells in vitro ${ }^{28}$. To evaluate whether in vivo RT might increase expression of KPNA2 in NSCLC we implanted a patient-derived tumor into mice, then exposed these tumors to one or three fractions of $8 \mathrm{~Gy}$ (the latter a regimen similar to the one used in patients). We found that tumor-derived KPNA2 gene expression was markedly upregulated at 24 hours (Fig. 4f).

Collectively these results demonstrate that RT in combination with CTLA-4 blockade induces systemic tumor responses in NSCLC patients. The early changes in TCR clonal dynamic seen in responders are consistent with a diversification and expansion of the tumordirected TCR repertoire induced by RT, as previously reported by us and others in preclinical tumor, models ${ }^{22,29}$. In depth analysis in representative patients suggests that expansion of a large number of tumor-specific $\mathrm{T}$ cell clones in peripheral blood, and their persistence over time correlate well with achievement of abscopal responses. 
Ipilimumab was similarly effective at activating the peripheral $\mathrm{T}$ cell compartment in responding and non-responding patients, while only responders showed a significant induction of IFN $\beta$. Thus, the different outcome might reflect RT ability to elicit the activation of a response in the tumor that mimics a viral infection. This interpretation is consistent with pre-clinical data demonstrating the requirement for RT-mediated induction of IFN $\beta$ via the cGAS/STING pathway for the activation of anti-tumor T cells ${ }^{4-6,30}$. The contribution of the cancer cells themselves to the production of IFN $\beta$ was also shown to be essential to achieve abscopal responses in combination with immune checkpoint inhibitors ${ }^{6,30}$. Patients with PD in this study show an impaired RT-induction of IFN $\beta$. While no mutations in cGAS and STING or other components of type I IFN signaling were found in patients \#36 and \#38, epigenetic downregulation of these genes, as recently shown in several tumors including lung cancer ${ }^{31}$, cannot be excluded. Although we did not have viable tumor tissue to test this hypothesis, it is possible that in some tumors the RT dose threshold for the induction of the exonuclease TREX1, which controls RT ability to induce the activation of cGAS by cytosolic DNA, is lower, explaining the failure to activate type I IFN $^{6}$.

Our most intriguing result was the identification of two $\mathrm{T}$ cell clones that are markedly expanded in the periphery or induced de novo in one patient with CR. Both clones recognize a mutation that was present in two separate brain metastases removed surgically before the patient entered the trial. The mutation-generated epitope is encoded in a gene, KPNA2, that is upregulated by RT. Linking all of our findings, it appears that, RT exposes immunogenic mutations by enhancing their expression in the tumor, followed by antigen crosspresentation and T cell activation inducing IFN $\beta$. Although to date, our complete analysis has been restricted to a single patient, we believe that these results may represent a paradigm underling the synergy of RT and CTLA-4 blockade resulting in the induction of a robust anti-tumor $\mathrm{T}$ cell response.

Recently, ipilimumab was shown to add efficacy to nivolumab (an anti-PD-1 agent) in metastatic chemotherapy-naïve NSCLC with high mutational burden ${ }^{32}$. The data presented here provide evidence for an additional role of ipilimumab in combination with RT in the treatment of metastatic NSCLC.

\section{METHODS}

\section{Study design}

NCT02221739 was a single institution pilot/feasibility trial of local radiotherapy and Ipilimumab in chemotherapy-refractory metastatic NSCLC patients. The study was designed to test the hypothesis that radiation to a metastatic site could convert it into an in situ vaccine and synergize with blockade of the CTLA-4 receptor to induce anti-tumor immune responses.

A Phase II clinical trial based on an optimum two-stage Phase II Simon design was used to conduct this pilot study. Ten patients were to be treated in Stage 1; in the absence of abscopal responses, the trial would terminate. With one or more abscopal responses in Stage 1 , the trial would proceed to enroll an additional 29 patients: a total of at least 4 abscopal 
responses $(10.25 \%)$ were needed to exclude futility. A radiation regimen of 6 Gy X 5 was tested for feasibility and safety during Phase I. In the absence of grade 4-5 toxicity, the study was designed to move to a radiation regimen of 9.5 Gy X 3 in Phase II. No other systemic or local agent was permitted during the course of the trial.

\section{Patients}

Eligible patients had to be aged 18 or older, have metastatic NSCLC and an Eastern Cooperative Oncology Group (ECOG) performance status of 2 or less, a life expectancy of $>3$ months, with adequate organ and marrow functional initial laboratory tests. Patients needed to have at least 2 distinct measurable metastatic sites. Patients with a previous history of brain metastasis were eligible if the brain metastases were controlled. A history of autoimmune disease excluded patients from eligibility to this trial. Similarly, any immunotherapy within 4 weeks from entering the study was a criterion for exclusion.

\section{Endpoints}

The primary endpoint of the study was the induction of immunity-mediated tumor response outside the radiation field (abscopal effect), as evidence of synergy of radiotherapy with Ipilimumab. Per protocol these measurements excluded the irradiated lesion and were conducted by both, immune related response criteria (irRC) ${ }^{33}$, and RECIST1.1 criteria to facilitate comparison with other studies and are reported consistently. Secondary endpoints were: 1) To determine if the clinical response was associated with an anti-tumor immune response and 2) To assess the toxicity of immunotherapy with radiation therapy in the tested regimens of dose and fractionation (6 Gy X 5 and 9.5 Gy X 3).

\section{Study oversight and ethical compliance}

The study protocol was approved by New York University and by Weill Cornell Medicine Institutional Review Boards, and the investigators complied with all ethical regulations pertaining to clinical research. All patients provided written informed consent before study entry. The study was deemed exempt from Investigational New Drug (IND) approval by the Food and Drugs Administration (FDA), exemption 122404. The Principal Investigator (SCF) was responsible for oversight of the clinical study.

Bristol Meyer Squibb provided free Ipilimumab for use in this study but did not have any role in the design, data collection and analysis, interpretation of results and preparation of the manuscript.

\section{Procedures}

Patients underwent baseline radiographic assessment by PET/CT within a week before first treatment to establish 2 measurable lesions and select one for radiotherapy. The radiotherapy regimen consisted of 6 Gy X 5 daily fractions in Phase I of the study, and 9.5 Gy X 3 in Phase II. Radiation was delivered by external beam with linear accelerator with imageguided radiotherapy (IGRT) or intensity modulated radiotherapy (IMRT) techniques.

All patients received intravenous Ipilimumab after the first radiation treatment, at a dose of 3 $\mathrm{mg} / \mathrm{kg}$. Ipilimumab was repeated every three weeks for four cycles, unless disease 
progression or death, unacceptable toxicity or patient decision to withdraw participation from the study. With the exception of radiation-associated mucositis and/or skin toxicity, the protocol required return to baseline of all grade 3-4 toxicities prior to the start of the next immunotherapy treatment. Dose modification for hematologic or gastro-intestinal toxicity were based on the worst toxicity observed during the prior dose. Specifically for $>$ Grade 3 diarrhea, immunotherapy was held until recovered to < Grade 1 . Ipilimumab-induced gastrointestinal toxicity was managed following established common guidelines (https:// www.fda.gov/downloads/Drugs/DrugSafety/ PostmarketDrugSafetyInformationforPatientsandProviders/UCM249435.pdf).

Assessment of response by PET/CT occurred at day 88 from first dose of Ipilimumab. Patients were evaluated for abscopal responses (tumor regression of non-irradiated lesions) with baseline and post-treatment (day 88) PET/CTs. Assessment excluded the irradiated site, both in baseline measurement and at day 88. Metastases were measured on axial CTs in 2 perpendicular dimensions, per protocol, following irRC. Specifically, the products of the measurements (length $\mathrm{x}$ width) in all measurable lesions outside the radiation field were summed at baseline and compared to the sum of the measurements of the same lesions and potential new lesions post-treatment. The difference represented the abscopal response. Abscopal responses were assessed by PET/CT at day 88 and reported as: $\mathrm{CR}$ - complete resolution, PR - decrease in size $250 \%$ (irRC) or $230 \%$ (RECIST), PD - increase in size $25 \%$ (irRC) or $\geq 20 \%$ or the appearance of new lesions (RECIST), and SD - insufficient shrinkage or growth to qualify for PR/CR or PD. Toxicities were reported according to the common terminology criteria for adverse (CTCAE) events version 4.0.

Serial blood samples were collected for serum, plasma, and peripheral blood mononuclear cells (PBMC) at baseline, on days 22, 43, 64, and 88, and when indicated, at follow-up visit. Viable PBMC was stored in liquid nitrogen. Serum and plasma samples were stored frozen in aliquots.

\section{Immunohistochemistry}

Tumor tissue was analyzed for Programmed cell death ligand 1 (PD-L1) expression by immunohistochemistry (IHC), performed on formalin-fixed, paraffin-embedded, 4- $\mu$ m tissue sections using unconjugated, rabbit anti-human PDL-1 (CD274) clone SP142 (PDL1, Spring Biosciences catalog number M4420) $)^{34}$. Staining was performed on a Ventana Medical Systems Discovery XT instrument with online deparaffinization and using Ventana's reagents and detection kits. PDL1 was antigen retrieved in Ventana Cell Conditioner 1 (TrisBorate-EDTA) for 20 minutes. Endogenous peroxidase activity was blocked with 3\% hydrogen peroxide for 4 minutes. PDL1 was diluted 1:50 in phosphate buffered saline and incubated for 60 minutes at 37C. Primary antibody was detected with hapten linked, antirabbit multimer incubated for 20 minutes followed by anti-hapten horseradish peroxidase conjugate for 20 minutes. The complex was visualized with 3,3 diaminobenzidene and enhanced with copper sulfate. A tissue microarray containing placental tissue was used as positive control. PDL-1 expression was quantified as percentage of tumor cells with positive membrane staining and intensity classified on a scale of 1 to 3. In some samples, CD8 T cell infiltration was assessed by staining with rabbit anti-human CD8 clone SP57 (Ventana 
Medical Systems catalog number 790-4460). The number of CD8 ${ }^{+}$intra-tumoral T cells was counted in 3 to 5 representative fields (magnification 200X). CD8 T cell infiltration was considered marked (average $>50$ cells/200X field), moderate (average 10-49 cells/200X filed), and minimal (average $<10$ cells/200X field).

\section{Galectin, IFN $\beta$, and sCD25 ELISA measurements}

We used enzyme-linked immunosorbent assay (ELISA) to measure serum levels of (a) sCD25, as a marker that has been shown to have prognostic value in patients with melanoma treated with ipilimumab ${ }^{17}$, (b) IFN $\beta$ that is mechanistically relevant to the immunogenicity of radiotherapy ${ }^{6}$, and (c) galectin-1, an immune suppressive tumor-secreted factor that can be increased by radiation ${ }^{16}$. Serum samples were stored at $-80 \mathrm{C}$ and thawed only once prior to analysis. Commercially available ELISA kits were used to determine serum levels of galectin-1 (Cat DGAL10, R\&D Systems), IFN $\beta$ (Cat 41415-1, PBL assay Science) and soluble CD25 (Cat BMS212INST, eBioscience) according to manufacturer's instructions with each sample run in triplicate wells. The mean minimum detectable concentrations for Gal-1, IFN $\beta$ and sCD25 are $0.022 \mathrm{ng} / \mathrm{mL}, 1.2 \mathrm{pg} / \mathrm{mL}, 0.21 \mathrm{ng} / \mathrm{ml}$, respectively.

\section{Analysis of antibodies specific for MICA/B}

MICA and MICB antibodies in human sera were analyzed with a fluorescence-based sandwich ELISA. Recombinant soluble proteins representing the MICA*008 or MICB*005 extracellular domains were incubated separately at $1 \mu \mathrm{g} / \mathrm{ml}$ in DELFIA ${ }^{\circledR}$ Yellow 96-wells Plates for $12-16$ hours at $4{ }^{\circ} \mathrm{C}$. The wells were then washed with TBS / $0.05 \%$ Tween-20 and blocked with DELFIA Assay Buffer (PerkinElmer, catalog 1244-111) for 2 hours at room temperature. Human serum samples were diluted 1:1000 in DELFIA Assay Buffer, added to the wells, and incubated for 2 hours at room temperature. The wells were subsequently washed with TBS / $0.05 \%$ Tween-20 and incubated for one hour at room temperature with DELFIA Eu-N1 Anti-Human IgG (PerkinElmer, catalog 1244-330) diluted 1:500 in DELFIA Assay Buffer. Wells were then washed with TBS / 0.05\% Tween-20 followed by addition of DELFIA Enhancement Solution (PerkinElmer, catalog 1244-105). Time-resolved europium fluorescence was analyzed using an EnVision ${ }^{\circledR}$ Plate Reader (PerkinElmer). Analyses were done in triplicates for each sample.

\section{Quantification of serum shed MICA and MICB}

Shed MICA and MICB in human sera were quantified using Human MICA ELISA Kit (AbCAM, ab59569) and DuoSet® Human MICB Kit (R\&D Systems, DY1599), respectively. The procedures were performed according to recommendations in the data sheets for both kits. Analyses were done in triplicates for each sample. We used recombinant soluble MICA*008 and $\mathrm{MICB} * 005$ proteins representing the extracellular domains (Baculovirus expression system) for the generation of standard curves because protein concentrations were overestimated when protein standard provided in the commercial kits were utilized. Human sera were analyzed in these assays after 1:5 dilution. Absorbance at $450 \mathrm{~nm}$ was analyzed in an EnVision® Plate Reader (PerkinElmer). 


\section{Flow cytometry analysis of PBMC}

Cryopreserved peripheral blood mononuclear cells (PBMC) were thawed and stained with fixable viability dye eFluor 450 (eBioscience) for $30 \mathrm{~min}$ at $4^{\circ} \mathrm{C}$. FcR-binding inhibitor (eBioscience) was added to all cells followed by incubation with antibodies targeting surface markers (see Life Sciences Reporting Summary for list of antibodies and dilutions used).

Prior to addition of antibodies targeting intracellular targets, cells were permeabilized and fixed using Fixation/Permeabilization Solution Kit (eBioscience). Samples were acquired on a MACSquant Analyser 10 (Miltenyi Biotec) and data was analyzed using the FlowJo software (Treestar). The gating strategy is illustrated in Supplementary Fig. 6.

\section{TCRB CDR3 sequencing and repertoire analysis}

DNA was isolated from PBMC using the DNeasy Blood \& Tissue Kit (Qiagen).

Amplification and sequencing of TCRB CDR3 regions was performed at Adaptive Biotechnologies (Seattle, WA) using their developed ImmunoSEQ platform ${ }^{35}$. The TCRB CDR3 regions in the sequencing reads were defined according to the ImMunoGeneTics (IMGT) collaboration. A standard algorithm was used to identify which V, D and J segments contributed to each TCRB CDR3 sequence ${ }^{36}$, and only in-frame TCR rearrangements were included in the analysis. For all samples, a standardized amount of DNA was used to amplify and sequence TCRB CDR3 regions. Several molecular and bioinformatic methods are used to control for PCR bias and ensure that the ImmunoSEQ assay is quantitative ${ }^{35}$. For every sample investigated, synthetic TCR genes are added and act as controls for bioinformatic normalization of amplification bias for every $\mathrm{V} \& \mathrm{~J}$ gene segment in the genome. These synthetic molecules also permit measuring the absolute cellular abundance of $\mathrm{T}$ cell clones, and analysis is not performed only on relative abundance between samples ${ }^{37}$.

TCRB CDR3 repertoire clonality was calculated as $1-$ Pielou's evenness $^{38}$. Normalization ensures estimation of clonality is robust to variations in sample size and enables assessment of relative degree of clonality between samples.

Expansion and contraction of $\mathrm{T}$ cell clones was determined using the Differential Abundance tool in the Adaptive Biotechnologies immunoSEQ analyzer ${ }^{39}$. Analysis settings: count $=$ nucleotide, minimum total filter $=10$, productive only $=$ TRUE; method $=$ binomial; alternative $=t w o-s i d e d$, multiple test correction $=b h$, and alpha (significance threshold) $=$ 0.01. Differential abundant clone analysis was performed in two ways. First, we determined the number of statistically significantly expanded or contracted clones between baseline and day 22 in PBMC for patients with TCR-seq data at these two time-points ( $\mathrm{n}=7,5$, and 8 for $\mathrm{CR} / \mathrm{PR}, \mathrm{SD}$, and PD, respectively). Statistical significance within and between groups was determined using a two-sided t-test (paired comparisons for within group analysis and assuming unequal variance for between group analysis). Secondly, we performed an indepth analysis of the TIL TCR dynamics for patients 4, 32, 36, and 38, for which we were able to perform TCR sequencing on pre-treatment tumor tissue. Clones with statistically significant expansion in PBMC from baseline during treatment were determined and classified as TILs if also present in $\geq 1$ tumor biopsy (patient 4 had two tumor tissue samples 
available). When plotting TCR frequency against time, frequencies were $\log _{10}$-transformed, and not detected clones were given a frequency of $10^{-6}$ (annotated as n.d. in figures).

The Needleman-Wunsch (NW) global alignment algorithm was used to calculate amino acid sequence similarity between TIL-TCRs CDR3 regions for patient 4 (previously described in 22,23 ). All work related to sequence similarity analysis was performed in R 3.4.3. A distance matrix based on NW similarity score was calculated between all TIL-TCRs $>0.1 \%$ in the tumor and not increased in blood together with the TIL-TCRs increased in blood during treatment and present in one or both tumor samples. The distance matrix was calculated using the score function within the Biostrings package (version 2.46.0); settings: type $=$ 'global', substitutionMatrix $=$ PAM10, gapOpening $=30$, gapExtension $=8)^{40}$. Then, the $N J$ function within the phangorn package (version 2.4.0) was used to construct a sequence similarity dendrogram ${ }^{41}$. The result was visualized as a weighted unrooted dendrograms using the ggtree function within the ggtree package (version 1.10.5) ${ }^{42}$. Area of circles represent frequency of a given TIL-TCR clone.

\section{Random forest classification and variable importance analysis}

Random forest classification was performed using the randomForestSRC package (version 2.5.1) in R 3.3.2 ${ }^{43}$. Patients' response was classified using variables with statistically significant difference when comparing day 22 and baseline for any response group. The $r f s r c$ function was executed in R with default parameters except for $n t r e e=2000$, tree.err $=T$, importance $=T$, and na.action $=$ 'na.impute'. Variable importance scores, classification error rates, and maximal subtree data was determined from 1000 executions of $r f s r c{ }^{44}$. For each variable, importance score average and standard deviation was calculated. For all random forest classification analysis, patient 33 was removed due to missing data for all variables except absolute lymphocyte counts (ALC). Additionally, missing flow cytometry for patients 17,23 , and 44 was imputed using the default missing data algorithm within the $\mathrm{R}$ randomForestSRC $r$ ssrc function ${ }^{45,46}$

\section{Whole exome sequencing}

DNA was isolated from formalin-fixed paraffin-embedded (FFPE) tumor blocks using the Qiagen QIAamp DNA FFPE Tissue kit (cat no 56404). Then, $250 \mathrm{ng}$ of DNA from each sample were sheared on a Covaris instrument for 360 seconds (Duty cycle - 10\%; intensity 5; cycles/Burst - 200). Barcoded libraries were prepared using the Kapa Low-Throughput Library Preparation Kit Standard (Kapa Biosystems), amplified using the KAPA HiFi Library Amplification kit (Kapa Biosystems) (8 cycles) and quantified using Qubit Fluorimetric Quantitation (Invitrogen) and Agilent Bioanalyzer. Two equimolar pool of the 6 and 5 barcoded libraries respectively, were used as input to capture the exome using one reaction tube each of the Nimblegen SeqCap EZ Human Exome Library v3.0 (Roche, cat \# 06465684001), according to the manufacturer's protocol. The pooled capture libraries were quantified by Qubit (Invitrogen) and Bioanalyzer (Agilent) and sequenced on an Illumina Illumina HiSeq 2500 using a paired end, 100 nucleotides in length run mode, across three lanes. The sequencing reads were cleaned by trimming adapter sequences and low-quality bases, and were aligned to the human reference genome (GRCh37) using BWA ${ }^{47}$. The 
aligned bam file was further refined by duplicate removal with Picard toolkit, and indel realignment and base quality recalibration with the Genome Analysis Toolkit ${ }^{48}$.

Baseline tumor and PBMC WES data was used in VarScan2 to detect somatic variants ${ }^{49}$. Variants with somatic p-value (SPV) $<0.05$ were considered statistically significant.

\section{RNA sequencing}

RNA was isolated from FFPE tumor blocks using the RNeasy FFPE kit (cat no 73504). Libraries were prepared using the Illumina TruSeq Stranded Total RNA library prep, after ribodepletion with Ribozero Gold kit (cat\# 20020597) starting from $800 \mathrm{ng}$ of DNAse I treated total RNA, following the manufacturer's protocol, with the exception that 10 cycles of PCR were performed to amplify the libraries, to keep the duplication rate lower than with the recommended 15 cycles. The amplified libraries were purified using AMPure beads, quantified by Qubit and QPCR, and visualized in an Agilent Bioanalyzer. The libraries were pooled equimolarly, and sequenced on an Illumina HiSeq 2500, v4 chemistry as paired end 50 . The sequencing reads were cleaned by trimming adapter sequences and low-quality bases, and then aligned to the human reference genome (GRCh37) using STAR ${ }^{50}$. Cufflinks was used to measure transcript abundances in Fragments Per Kilobase of exon model per Million mapped reads (FPKM) ${ }^{51,52}$.

\section{Neoantigen predictions}

To predict patient-specific neoantigens, HLA-typing was performed from WES data using Optitype, a HLA genotyping algorithm (Supplementary Table 8 ) $^{53}$. Before predicting, VCF files containing somatic mutations were subjected to standard VEP, Downstream, and Wildtype annotations, and filtered for frameshift and missense mutations ${ }^{54}$. Additionally, variant allele frequency (VAF) data was generated using bamreadcount (https://github.com/ genome/bam-readcount). The NetMHC and NetMHCpan algorithms were used to predict patient-specific MHC-I binding for all possible 8-11 aa long variant and corresponding wildtype peptide chains spanning the mutation using the pVAC-seq pipeline ${ }^{55-57}$.

When comparing neoantigen load between patients $4,32,36$, and 38 , a neoantigen was defined as a variant peptide binding MHC-I with an affinity $<500 \mathrm{nM}$ passing the following filters: normal tissue WES site coverage $>5$, normal tissue VAF $<3$, tumor tissue WES site coverage $>5$, tumor tissue VAF $>10$. Additionally, a Lung Adenocarcinoma gene expression dataset was used to determine median RNA gene expression for each gene encoding a predicted neoepitope ${ }^{58}$.

For patient 4 (CR), baseline tumor tissue was sufficient to perform RNA-seq in addition to WES. Gene expression levels were calculated using cufflinks and RNA-seq VAF data was determined using bamreadcount ${ }^{51}$. Then, variant peptides for this patient were further filtered on baseline tumor gene expression ( $>1$ FPKM) and RNA-seq variant allele frequency $(>0 \%)$. 


\section{T cell response to neoantigens}

The 33 peptides that qualified as potential neoantigens were synthesized at a purity exceeding $85 \%$ (Genscript), dissolved in DMSO $(5 \mathrm{mg} / \mathrm{mL})$ and tested in vitro for recognition by the patient \#4 T cells from post-treatment blood samples. PBMC were thawed and cultured overnight with the peptides (final concentration: $2 \mu \mathrm{g} / \mathrm{mL}$ ) in RPMI medium (Gibco) containing 5\% human AB serum (Sigma-Aldrich, H3667), 30 IU/mL IL2 (Miltenyi Biotec, 130-097-745), 20 IU/mL IL7 (Miltenyi Biotec, 130-093-937), 10 IU/mL IL15 (Miltenyi Biotec, 130-093-955) and 1x non-essential amino acids (Sigma-Aldrich, M7145) in a 96-well round-bottom plate. Peptides were first tested as pools (33 peptides in 9 pools) in comparison with DMSO treatment alone. Brefeldin A (Thermo Fisher Scientific, 00-4506-51) was added $1 \mathrm{~h}$ after adding the peptides to the culture. After overnight incubation, cells were washed and stained with the viability dye eFluor ${ }^{\circledR} 450$ (Thermo Fischer Scientific, 65-0863-14) and with CD3-APC and CD8-PerCP-vio700 ${ }^{\mathrm{TM}}$ antibodies (Miltenyi Biotec, 130-091-373 and 130-097-911 respectively). Subsequently, cells were fixed and permeabilized (Thermo Fisher Scientific, 88-8824-00) before intracellular staining of IFN $\gamma$-FITC (Miltenyi Biotec, 130-091-641). Samples were analyzed on a MACSQuant flow cytometer (Miltenyi Biotec) and data were analyzed using FlowJo software. Peptides selected from the positive pools were then tested individually using the same procedure. In this experiment, reactivity of healthy donor $\mathrm{CD}^{+} \mathrm{PBMC}$ against the same peptides was assessed, demonstrating a lack of background/non-specific staining.

Patient's PBMC reactivity towards selected peptides was then confirmed by measuring IFN$\gamma$ production using a standard overnight enzyme-linked immunosorbent spot (ELISpot) assay. Briefly, 96-well nitrocellulose plates (Millipore Sigma, MSHAS4510) were coated with anti-IFN- $\gamma$ monoclonal antibody $(4 \mu \mathrm{g} / \mathrm{ml})$ and incubated overnight at $4{ }^{\circ} \mathrm{C}$. Plates were washed and blocked with RPMI medium supplemented with $10 \%$ human serum for $2 \mathrm{~h}$ at $37^{\circ} \mathrm{C}$. T cells stimulated for 20 days with the test peptide were added to wells in duplicate at 25,000 cells or 5,000 CD8 ${ }^{+}$cells per well and were stimulated overnight with autologous BAPCs loaded with mutated peptides or their wild type counterpart $(2 \mu \mathrm{g} / \mathrm{mL})$. Stimulation with PMA/ionomycin was used as positive control for T cell reactivity. After 18h, the ELISpot plate was washed and processed for spot development following manufacturer's instructions. Spots were counted using an automated ELISpot plate reader (CTL ImmunoSpot, Cellular Technology Limited). Empirically, we consider that four times the spot number in the $[5,000]$ well is equivalent to the spot number in $[25,000]$. Means were calculated as follows: average $([25,000], 4 *[5,000])$. Peptide-stimulated responses were considered positive if the mean $>50$ spots (positive cut-off).

\section{Gene expression analysis using qRT-PCR}

Patient-derived tumor xenografts (PDTX) from freshly resected primary lung tumor were implanted in NOD/SCID/gamma (NOG) female mice (CIEA NOG mouse; NOD.CgPrkdc ${ }^{\text {scid }}$ II2rg ${ }^{\text {tmlSug} / J i c T a c, ~ T a c o n i c ~ A n i m a l ~ L a b o r a t o r y, ~ G e r m a n t o w n, ~ N Y) ~ a n d ~ i r r a d i a t e d ~}$ as previously described ${ }^{6}$. All experiments were approved by the Institutional Animal Care and Use Committee. Total RNA was extracted from frozen tumor tissues using RNeasy Plus Mini Kit (QIAGEN, 74134) according to the manufacturer's instructions. Complementary cDNA synthesis was performed using the SuperScript ${ }^{\mathrm{TM}}$ IV VILO Master mix (Thermo 
Fischer Scientific, 11756500) according to the manufacturer's instructions. Quantitative polymerase chain reaction was performed using the iTaq Universal SYBR Green Supermix (Biorad, 172-5121) and the primers for human KPNA2 (Biorad, assay ID: qHsaCED0047532) and RPL13 (Biorad, assay ID: qHsaCED0020417). All samples were carried out in triplicate in a MicroAmp Optical 96-well reaction plate (Applied Biosystems), and a negative control with no cDNA template was included in every run. The reaction was conducted in the Applied Biosystems 7500 real-time PCR cycler (ThermoFisher) with amplification under the following conditions: $95^{\circ} \mathrm{C}$ for $10 \mathrm{~min}$, followed by 40 cycles of $95^{\circ} \mathrm{C}$ for $15 \mathrm{~s}$ and $60^{\circ} \mathrm{C}$ for $1 \mathrm{~min}$. A melting curve analysis was also done to ensure the specificity of the corresponding RT-PCR reactions. The relative expression of the gene was determined with the $2^{-\mathrm{DDCt}}$ method, with RPL13 as housekeeping gene.

\section{Statistical analysis}

All time-to-event endpoints were summarized using the Kaplan-Meier method. Difference in these endpoints between groups of interest were examined using a log-rank test. These analyses were carried out among the intention-to-treat population. A two-sided p-value $<0.05$ was considered statistically significant.

The study was not powered to detect the impact of RT site or RT regimen on treatment response. Nevertheless, this was tested using Chi-square (for RT site) and Fisher exact (for RT regimen, 6 Gy X 5 versus 9.5 Gy X 3) tests and no significant associations were found.

For continuous baseline measurements, differences across response groups were examined using the non-parametric Kruskal-Wallis test. For a continuous biomarker measured over time, a linear mixed-effects model with random intercept was used to estimate the measurement at different time points for each treatment groups. This model takes into account both the between and within subject variances. The levels of a few biomarkers were square root transformed prior to analysis to ensure the underlying model assumptions were satisfied (indicated in the results of each biomarker). Differences in measurements between day 22 and baseline for the response groups were subsequently examined using simultaneous tests for general linear hypotheses. Linear mixed-effects models and simultaneous tests for general linear hypotheses were also used to examine the association between the change between baseline and maximum/minimum post-treatment levels of each biomarker and the response groups. The outcome variable for each of the model was the baseline and the post treatment maximum/minimum biomarker levels and the fixed effects covariates included post-treatment status, response category, and their interactions. Due to the exploratory nature of the biomarker analysis and the limited sample size, no multiple testing adjustment was applied. P-value $<0.05$ is considered as statistically significant. Statistical analysis was carried out using R 3.3.1 (R Foundation for Statistical Computing, Vienna, Austria).

All statistical tests performed are two-sided.

\section{Data Availability.}

The data reported are tabulated in the manuscript and Supplementary figures and tables, and Supplementary Dataset 2 and 3. Raw data for soluble markers and flow cytometry are 
available in Supplementary Dataset 1. The raw TCR sequence data have been deposited into the ImmuneACCESS project repository of the Adaptive Biotechnology database (doi: 10.21417/B7BW6X). WES and RNAseq data have been deposited at the NCBI Sequence Read Archive (Accession number SRP136187; http://www.ncbi.nlm.nih.gov/bioproject/ 439205).

\section{Supplementary Material}

Refer to Web version on PubMed Central for supplementary material.

\section{Acknowledgments:}

We would like to acknowledge Dr. Judith Goldberg for the initial design of the clinical trial, Dr. Karsten Pilones for assistance with DNA preparation, Dr. Luis Chriboga for help with immunohistochemistry, Dr. Debra Morrison for blood processing, and the NYULH Genome Technology Center (GTC) technical personnel for sequencing. We thank Sharanya Chandraseckhar for data managing, Maria Fenton-Kerimian, NP, for patient care, and Dr. Giorgio Inghirami for providing the PDX mice. We thank Bristol Meyer Squibb, New York, NY, USA, for providing ipilimumab for this research study. We are indebted to Dr. Gary Koretzky for insightful discussion and review of the manuscript.

The immunological studies were funded by NCI R01CA198533 and R01CA201246 (SD). The NYU Experimental Pathology Immunohistochemistry Core Laboratory and the GTC are partially supported by the Cancer Center Support Grant P30CA016087 at the Laura and Isaac Perlmutter Cancer Center, NYULH. EW is supported by DOD W81XWH-17-1-0029 post-doctoral fellowship. SG acknowledges the Human Immune Monitoring Center at Mount Sinai and Cancer Center Support Grant P30CA196521. LFA was funded by a Friends for Life Neuroblastoma Fellowship, KWW was supported by NCI R01 CA173750.

\section{References}

1. Formenti SC \& Demaria S Systemic effects of local radiotherapy. Lancet Oncol 10, 718-726 (2009). [PubMed: 19573801]

2. Demaria S, Coleman CN \& Formenti SC Radiotherapy: Changing the Game in Immunotherapy. Trends Cancer 2, 286-294 (2016). [PubMed: 27774519]

3. Postow MA, et al. Immunologic correlates of the abscopal effect in a patient with melanoma. N Engl J Med 366, 925-931 (2012). [PubMed: 22397654]

4. Burnette BC, et al. The efficacy of radiotherapy relies upon induction of type i interferon-dependent innate and adaptive immunity. Cancer Res 71, 2488-2496 (2011). [PubMed: 21300764]

5. Deng L, et al. STING-Dependent Cytosolic DNA Sensing Promotes Radiation-Induced Type I Interferon-Dependent Antitumor Immunity in Immunogenic Tumors. Immunity 41, 843-852 (2014). [PubMed: 25517616]

6. Vanpouille-Box C, et al. DNA exonuclease Trex1 regulates radiotherapy-induced tumour immunogenicity. Nat Commun 8, 15618 (2017).

7. Zatloukal P, et al. Randomized phase II clinical trial comparing tremelimumab (CP-675, 206) with best supportive care (BSC) following first-line platinum-based therapy in patients (pts) with advanced non-small cell lung cancer (NSCLC). J Clin Oncol 27, 8071 (2009).

8. Lynch TJ, et al. Ipilimumab in combination with paclitaxel and carboplatin as first-line treatment in stage IIIB/IV non-small-cell lung cancer: results from a randomized, double-blind, multicenter phase II study. J Clin Oncol 30, 2046-2054 (2012). [PubMed: 22547592]

9. Golden EB, Demaria S, Schiff PB, Chachoua A \& Formenti SC An abscopal response to radiation and ipilimumab in a patient with metastatic non-small cell lung cancer. Cancer Immunol Res 1, 365-372 (2013). [PubMed: 24563870]

10. Golden EB, et al. Local radiotherapy and granulocyte-macrophage colony-stimulating factor to generate abscopal responses in patients with metastatic solid tumours: a proof-of-principle trial. Lancet Oncol 16, 795-803 (2015). [PubMed: 26095785] 
11. Spigel DR, et al. Total mutation burden (TMB) in lung cancer (LC) and relationship with response to PD-1/PD-L1 targeted therapies. J Clin Oncol 34, 9017-9017 (2016).

12. Gainor JF, et al. EGFR Mutations and ALK Rearrangements Are Associated with Low Response Rates to PD-1 Pathway Blockade in Non-Small Cell Lung Cancer: A Retrospective Analysis. Clin Cancer Res 22, 4585-4593 (2016). [PubMed: 27225694]

13. Ng Tang D, et al. Increased frequency of ICOS+ CD4 T cells as a pharmacodynamic biomarker for anti-CTLA-4 therapy. Cancer Immunol Res 1, 229-234 (2013). [PubMed: 24777852]

14. Wang W, et al. Biomarkers on melanoma patient T cells associated with ipilimumab treatment. J Transl Med 10, 146 (2012). [PubMed: 22788688]

15. Sim GC, et al. IL-2 therapy promotes suppressive ICOS+ Treg expansion in melanoma patients. J Clin Invest 124, 99-110 (2014). [PubMed: 24292706]

16. Kuo P, et al. Galectin-1 mediates radiation-related lymphopenia and attenuates NSCLC radiation response. Clin Cancer Res 20, 5558-5569 (2014). [PubMed: 25189484]

17. Koguchi Y, et al. Serum Immunoregulatory Proteins as Predictors of Overall Survival of Metastatic Melanoma Patients Treated with Ipilimumab. Cancer Res 75, 5084-5092 (2015). [PubMed: 26627641]

18. Jinushi M, Hodi FS \& Dranoff G Therapy-induced antibodies to MHC class I chain-related protein A antagonize immune suppression and stimulate antitumor cytotoxicity. Proc Natl Acad Sci U S A 103, 9190-9195 (2006). [PubMed: 16754847]

19. Ferrari de Andrade L, et al. Antibody-mediated inhibition of MICA and MICB shedding promotes NK cell-driven tumor immunity. Science In press(2018).

20. Gasser S, Orsulic S, Brown EJ \& Raulet DH The DNA damage pathway regulates innate immune system ligands of the NKG2D receptor. Nature 436, 1186-1190 (2005). [PubMed: 15995699]

21. Ruocco MG, et al. Suppressing T cell motility induced by anti-CTLA-4 monotherapy improves antitumor effects. J Clin Invest 122, 3718-3730 (2012). [PubMed: 22945631]

22. Rudqvist NP, et al. Radiotherapy and CTLA-4 Blockade Shape the TCR Repertoire of TumorInfiltrating T Cells. Cancer Immunol Res 6, 139-150 (2018). [PubMed: 29180535]

23. Sidhom JW, et al. ImmunoMap: A Bioinformatics Tool for T-cell Repertoire Analysis. Cancer Immunol Res 6, 151-162 (2018). [PubMed: 29263161]

24. Rizvi NA, et al. Cancer immunology. Mutational landscape determines sensitivity to PD-1 blockade in non-small cell lung cancer. Science 348, 124-128 (2015). [PubMed: 25765070]

25. Snyder A, et al. Genetic basis for clinical response to CTLA-4 blockade in melanoma. N Engl J Med 371, 2189-2199 (2014). [PubMed: 25409260]

26. Van Allen EM, et al. Genomic correlates of response to CTLA-4 blockade in metastatic melanoma. Science 350, 207-211 (2015). [PubMed: 26359337]

27. Sharma P, Hu-Lieskovan S, Wargo JA \& Ribas A Primary, Adaptive, and Acquired Resistance to Cancer Immunotherapy. Cell 168, 707-723 (2017). [PubMed: 28187290]

28. Song KH, et al. Induction of immunogenic cell death by radiation-upregulated karyopherin alpha 2 in vitro. Eur J Cell Biol 95, 219-227 (2016). [PubMed: 27107455]

29. Twyman-Saint Victor C, et al. Radiation and dual checkpoint blockade activate non-redundant immune mechanisms in cancer. Nature 520, 373-377 (2015). [PubMed: 25754329]

30. Harding SM, et al. Mitotic progression following DNA damage enables pattern recognition within micronuclei. Nature 548, 466-470 (2017). [PubMed: 28759889]

31. Konno H, et al. Suppression of STING signaling through epigenetic silencing and missense mutation impedes DNA damage mediated cytokine production. Oncogene 37, 2037-2051 (2018). [PubMed: 29367762]

32. Hellmann MD, et al. Nivolumab plus Ipilimumab in Lung Cancer with a High Tumor Mutational Burden. N Engl J Med 378, 2093-2104 (2018). [PubMed: 29658845]

33. Wolchok JD, et al. Guidelines for the evaluation of immune therapy activity in solid tumors: immune-related response criteria. Clin Cancer Res 15, 7412-7420 (2009). [PubMed: 19934295]

34. Formenti SC, et al. Results of a phase I-II study of adjuvant concurrent carboplatin and accelerated radiotherapy for triple negative breast cancer. Oncoimmunology 6, e1274479 (2017). [PubMed: 28405497] 
35. Carlson CS, et al. Using synthetic templates to design an unbiased multiplex PCR assay. Nat Commun 4, 2680 (2013). [PubMed: 24157944]

36. Yousfi Monod M, Giudicelli V, Chaume D \& Lefranc MP IMGT/JunctionAnalysis: the first tool for the analysis of the immunoglobulin and T cell receptor complex V-J and V-D-J JUNCTIONs. Bioinformatics 20 Suppl 1, i379-385 (2004). [PubMed: 15262823]

37. Wu D, et al. Detection of minimal residual disease in B lymphoblastic leukemia by highthroughput sequencing of IGH. Clin Cancer Res 20, 4540-4548 (2014). [PubMed: 24970842]

38. Sherwood AM, et al. Tumor-infiltrating lymphocytes in colorectal tumors display a diversity of $\mathrm{T}$ cell receptor sequences that differ from the $\mathrm{T}$ cells in adjacent mucosal tissue. Cancer immunology, immunotherapy : CII 62, 1453-1461 (2013). [PubMed: 23771160]

39. DeWitt WS, et al. Dynamics of the cytotoxic T cell response to a model of acute viral infection. J Virol 89, 4517-4526 (2015). [PubMed: 25653453]

40. Pages H, Aboyoun P, Gentleman R \& DebRoy S Biostrings: Efficient manipulation of biological strings. R package version 2.46.0. (2017).

41. Schliep KP phangorn: phylogenetic analysis in R. Bioinformatics 27, 592-593 (2011). [PubMed: 21169378]

42. Yu G, Smith DK, Zhu H, Guan Y \& Lam TTY ggtree: an R package for visualization and annotation of phylogenetic trees with their covariates and other associated data. Methods in Ecology and Evolution 8, 28-36 (2017).

43. Ishwaran H \& Kogalur U Random Forests for Survival, Regression and Classification (RF-SRC). 2016. R package version 2.4. 1. (2017).

44. Ishwaran H Variable importance in binary regression trees and forests. Electron J Stat 1, 519-537 (2007).

45. Tang F \& Ishwaran H Random forest missing data algorithms. Statistical Analysis and Data Mining: The ASA Data Science Journal 10, 363-377 (2017).

46. Ishwaran H, Kogalur UB, Blackstone EH \& Lauer MS Random survival forests. The annals of applied statistics, 841-860 (2008).

47. Li, H. Aligning sequence reads, clone sequences and assembly contigs with BWA-MEM. arXiv preprint arXiv:1303.3997 (2013).

48. McKenna A, et al. The Genome Analysis Toolkit: a MapReduce framework for analyzing nextgeneration DNA sequencing data. Genome Res 20, 1297-1303 (2010). [PubMed: 20644199]

49. Koboldt DC, et al. VarScan 2: somatic mutation and copy number alteration discovery in cancer by exome sequencing. Genome Res 22, 568-576 (2012). [PubMed: 22300766]

50. Dobin A, et al. STAR: ultrafast universal RNA-seq aligner. Bioinformatics 29, 15-21 (2013). [PubMed: 23104886]

51. Trapnell C, et al. Transcript assembly and quantification by RNA-Seq reveals unannotated transcripts and isoform switching during cell differentiation. Nat Biotechnol 28, 511-515 (2010). [PubMed: 20436464]

52. Roberts A, Trapnell C, Donaghey J, Rinn JL \& Pachter L Improving RNA-Seq expression estimates by correcting for fragment bias. Genome Biol 12, R22 (2011). [PubMed: 21410973]

53. Szolek A, et al. OptiType: precision HLA typing from next-generation sequencing data. Bioinformatics 30, 3310-3316 (2014). [PubMed: 25143287]

54. McLaren W, et al. The Ensembl Variant Effect Predictor. Genome Biol 17, 122 (2016). [PubMed: 27268795]

55. Hundal J, et al. pVAC-Seq: A genome-guided in silico approach to identifying tumor neoantigens. Genome Med 8, 11 (2016). [PubMed: 26825632]

56. Nielsen M, et al. Reliable prediction of T-cell epitopes using neural networks with novel sequence representations. Protein Sci 12, 1007-1017 (2003). [PubMed: 12717023]

57. Andreatta M \& Nielsen M Gapped sequence alignment using artificial neural networks: application to the MHC class I system. Bioinformatics 32, 511-517 (2016). [PubMed: 26515819]

58. Cancer Genome Atlas Research, N. Comprehensive molecular profiling of lung adenocarcinoma. Nature 511, 543-550 (2014). [PubMed: 25079552] 


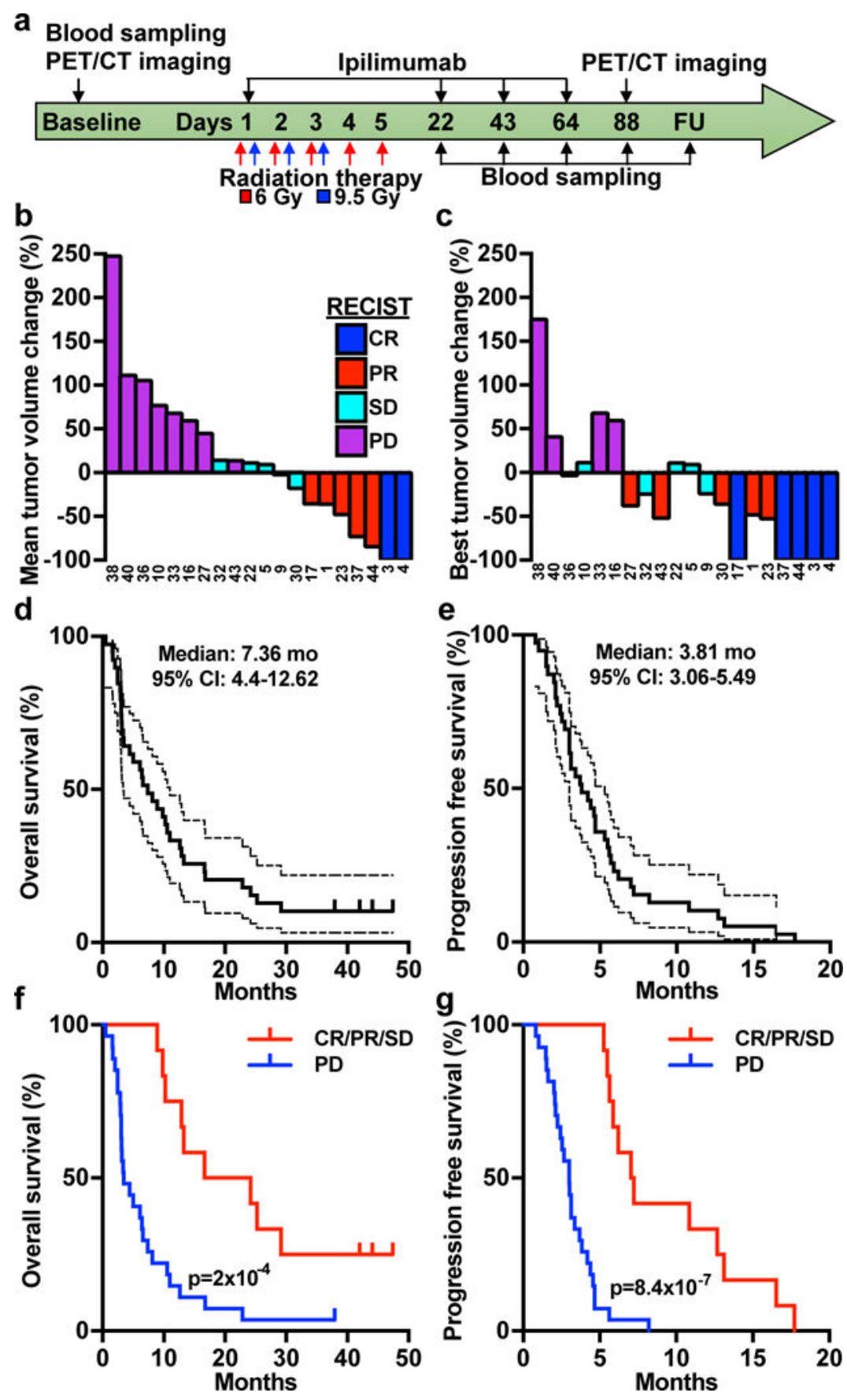

Figure 1. Patients survival and clinical response to radiotherapy and ipilimumab.

(a) Treatment, imaging, and blood sampling schema (FU: follow up). (b) Waterfall plot of aggregate tumor volume change in all non-irradiated lesions. Numbers at the bottom indicate patient ID\#. Patient 43 was classified as PD due to a new lesion. One patient had lesions that could not be accurately measured radiographically and is not included in the graph, but was considered as PD due to new lesions. (c) Best tumor volume change indicates the tumor volume change in the non-irradiated metastasis with the biggest change from baseline in each patient. Kaplan-Meier estimates of (d) overall survival and (e) progression-free survival 
for all patients $(\mathrm{n}=39)$. Comparison of $(\mathbf{f})$ overall and $(\mathbf{g})$ progression free survival between patients with disease control $(\mathrm{CR}+\mathrm{PR}+\mathrm{SD} ; \mathrm{n}=12)$ and with $\mathrm{PD}(\mathrm{n}=27)$. Overall survival was 20.4 (95\% CI: 12.9-not reached) and 3.5 (95\% CI: 3.1-7.4) months for CR/PR/SD and PD, respectively. Progression free survival was 7.1 (95\% CI: 5.9-not reached) and 3.0 (95\% CI: 2.4-3.8) months for CR/PR/SD and PD, respectively. Statistical significance was determined using a two-sided log-rank test. 

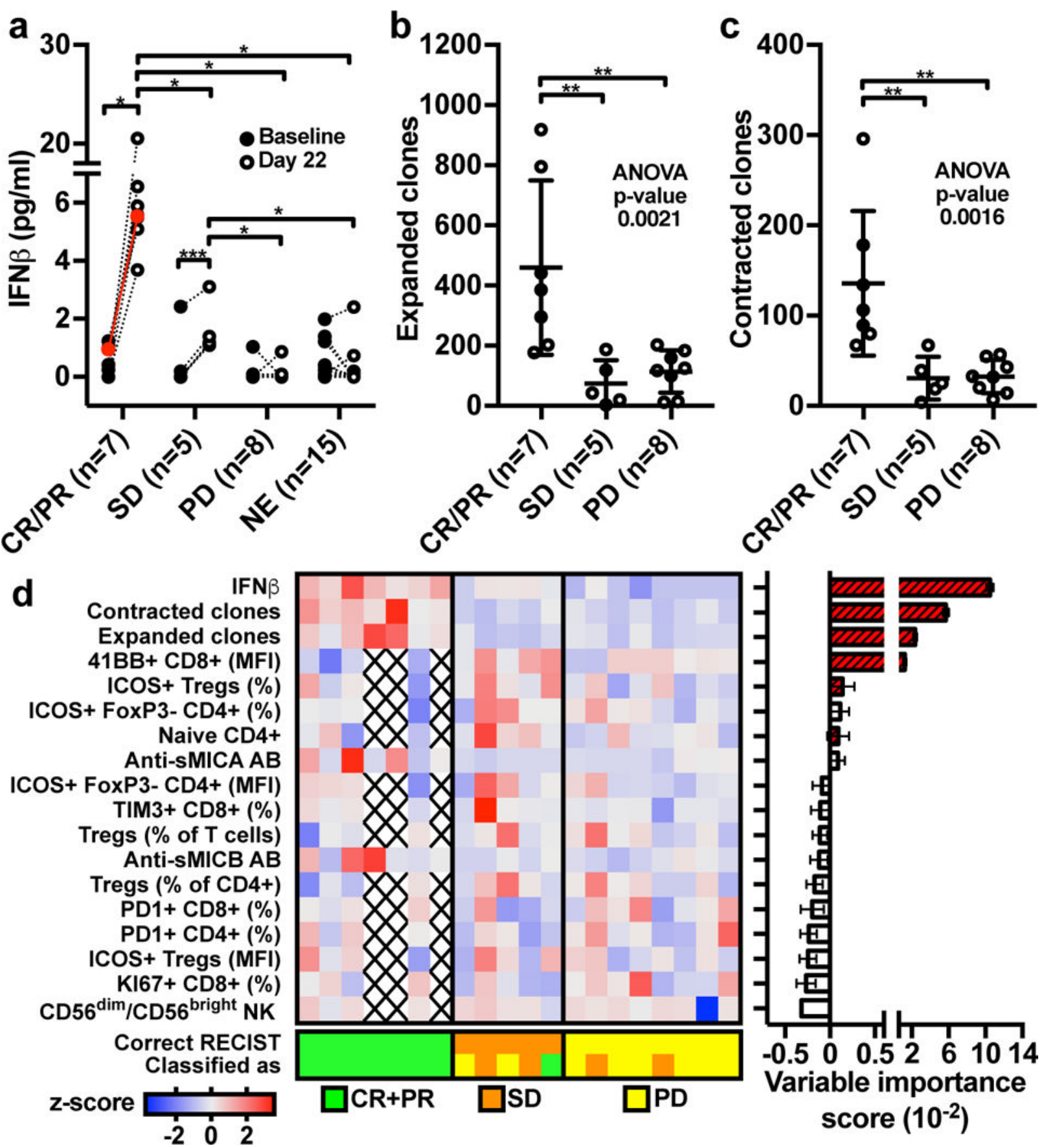

Figure 2. Increase in interferon- $\beta$ levels and TCR clonal dynamics predict response to treatment. (a) IFN $\beta$ serum levels at baseline (filled circles) and 22 days after treatment start (empty circle). The values for patient\#4 are indicated in red. NE indicates non evaluable patients. T cell receptor (TCR) sequencing was performed on peripheral blood at baseline and day 22 . Number of (b) expanded and (c) contracted TCR clones at day 22 compared to baseline. For panels $\mathrm{a}$, and $\mathrm{b}$ and c, statistical significance was determined using the two-sided Student's ttest and one-way ANOVA, respectively (p-values: $*<0.05, * *<0.01$, ***< 0.001 , and $* * * *<0.0001)$. Circles, horizontal lines, and error bars represent data-points for individual 
patients, means, and standard deviations, respectively. (d) Random Forest (RF) classification of patients using variables with statistically significant difference between day 22 and baseline for any RECIST response group (Supplementary Dataset 2). For IFN $\beta$, expanded and contracted clones, and anti-sMICA and sMICB antibodies (AB) measurements, $\mathrm{n}=7,5$, and 8 patients for $\mathrm{CR} / \mathrm{PR}, \mathrm{SD}$, and $\mathrm{PD}$ response groups, respectively. For all other measurements, $\mathrm{n}=4,5$, and 8 patients for $\mathrm{CR} / \mathrm{PR}, \mathrm{SD}$, and $\mathrm{PD}$ response groups, respectively. Performance of the RF classifications is shown under the heat-map. 'Correct RECIST' indicates the actual treatment response of each patient whereas 'Classified as' indicates how patients were classified using RF modeling. Additionally, mean and standard deviations of variable importance scores were determined (top predictive variables: red bars with filled pattern) from 1000 executions. 

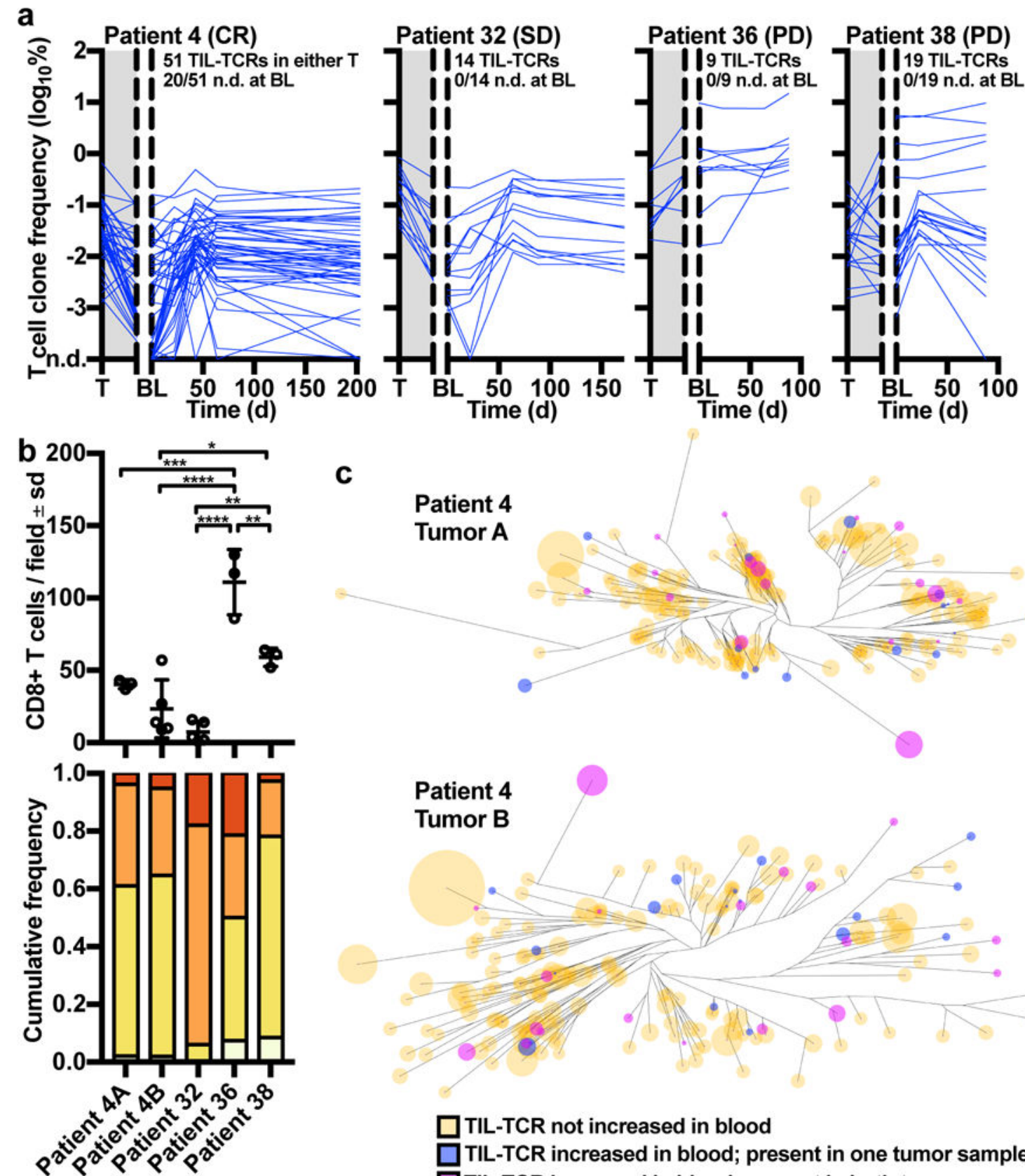

C
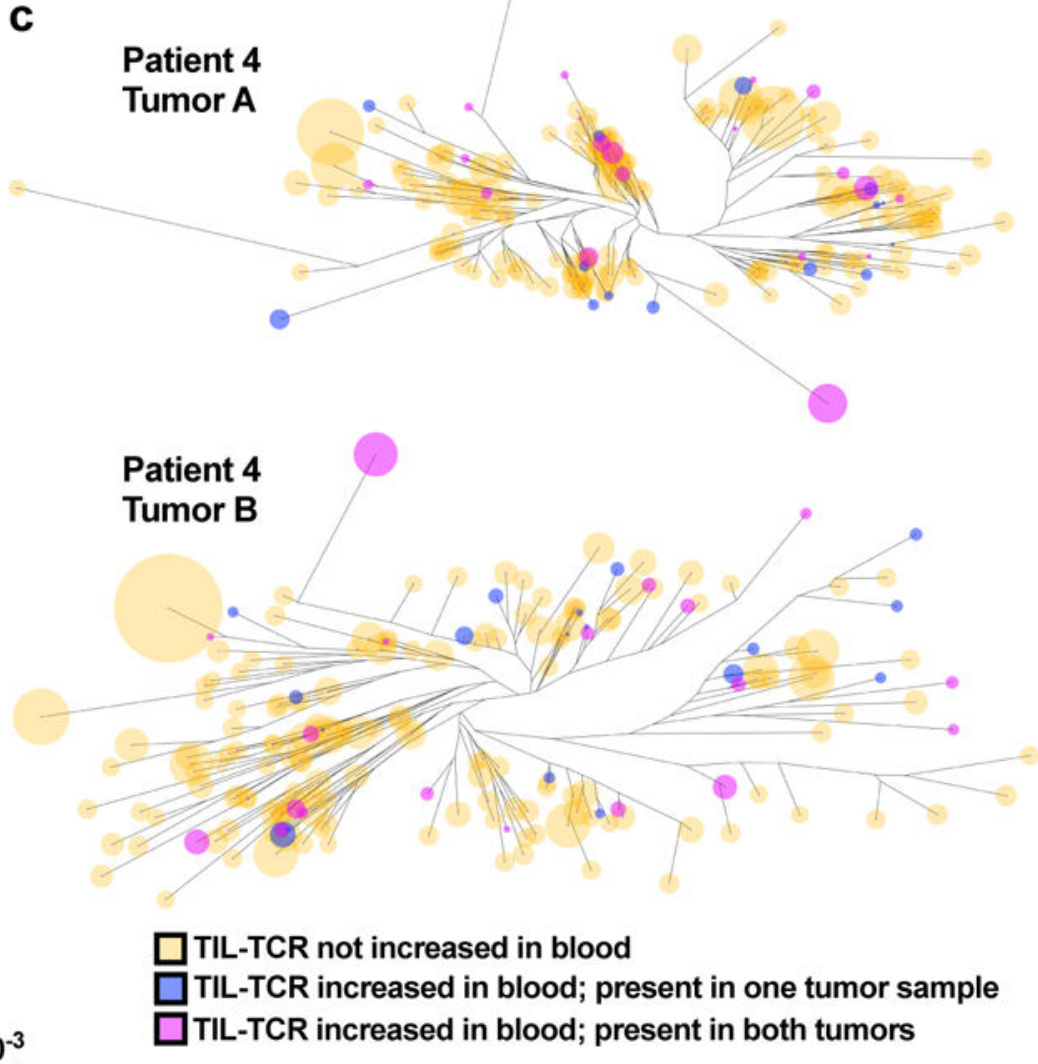

$\square 10^{-2}<\mathrm{f} \leq 10^{-1} \square 10^{-4}<\mathrm{f} \leq 10^{-3}$

$10^{-3}<\mathrm{f} \leq 10^{-2} \square 10^{-5}<\mathrm{f} \leq 10^{-4}$

Figure 3. Expansion of tumor-derived TCR clones in peripheral blood after treatment with radiotherapy and Ipilimumab.

(a) TCR-seq was performed on tumor (T) and blood of patient \#4 with CR, patient \#32 with SD, and patients \#36 and 38 with PD. TCR clones shown were present in tumor and significantly expanded in blood from baseline at any of the time points (day 22, day 43, day 64) during treatment. Each line represents a single TCR clone. n.d.: not detected. (b) Top graph: number of intra-tumoral $\mathrm{CD}^{+} \mathrm{T}$ cells per $200 \mathrm{X}$ field as determined by immunohistochemistry. Circles, horizontal lines, and error bars represent data-points, means, 
and standard deviations, respectively. $\mathrm{N}=3$ fields for patients 4 (tumor $\mathrm{A}$ ), 36 , and $38 . \mathrm{N}=5$ fields for patients 4 (tumor B) and 32. One-way ANOVA with Tukey's multiple comparisons test was used to determine statistical significance (p-values: $*<0.05$, $* *<0.01$, $* * *<$ 0.001 , and $* * * *<0.0001$ ). Bottom graph: Frequency space occupied by TCR clones of different sizes. Colors indicate size of TCR clones. (c) Sequence similarity of TCRs with frequency $>0.1 \%$ in the two pre-treatment brain metastases of patient \#4. A distance matrix based on Needleman-Wunch (NW) similarity score was calculated for TCRs not increased in blood (yellow) and TCRs increased in blood during treatment and present in one (blue) or both (purple) tumors. The Neighbor Joining method was used to construct TCR $\beta$ CDR3 sequence similarity dendrograms, visualized as unrooted dendrograms. Area of circles represents frequency of the TCR clone. 

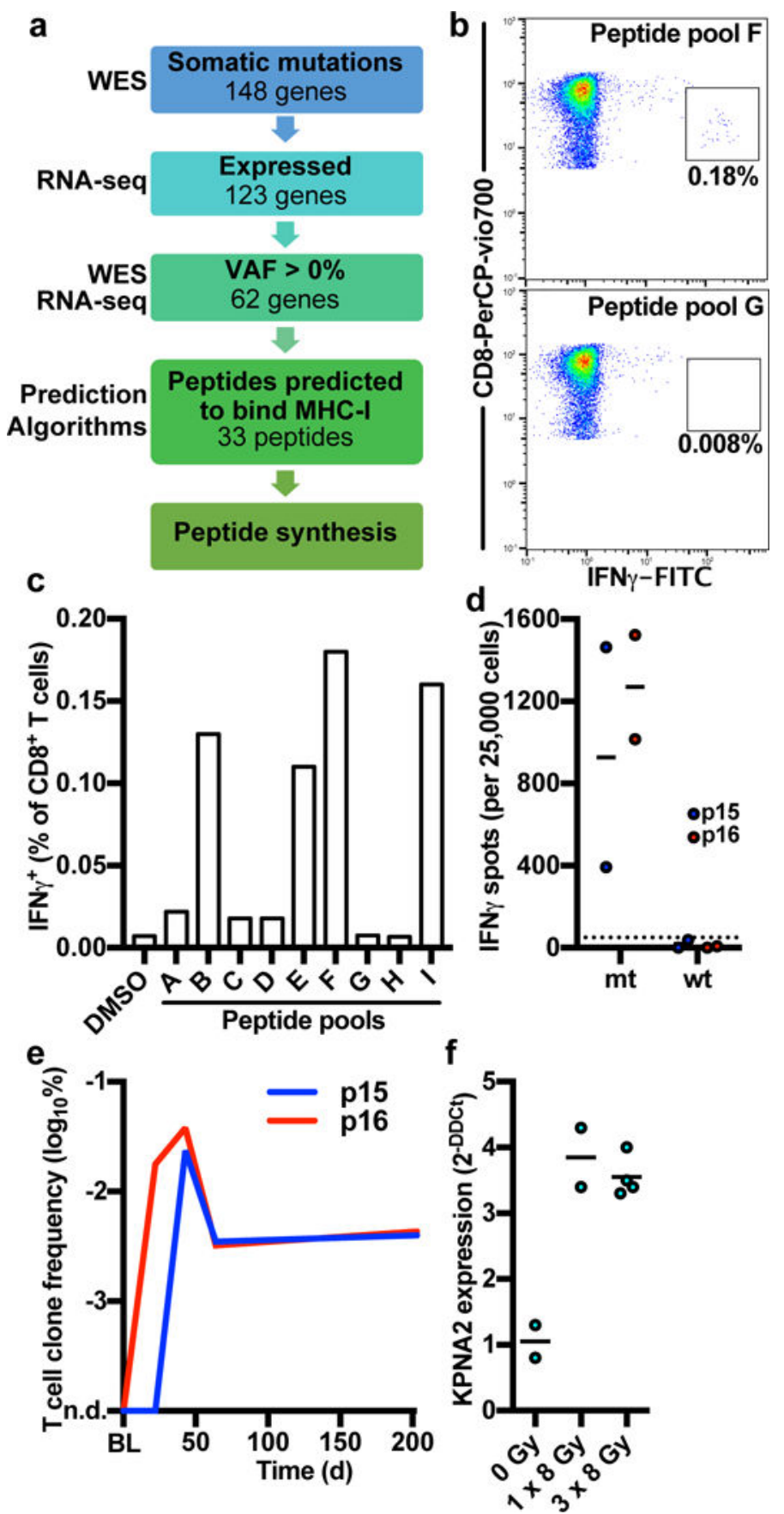

Figure 4. Expansion of neoantigen-reactive CD8 T cells in a NSCLC patient with complete response to radiotherapy and ipilimumab.

(a) Pipeline for neoantigen prediction. The somatic mutations identified by whole exome sequencing (WES) were filtered for expression of the mutated gene by RNAseq, variant allele frequency (VAF; variant reads/total reads) $>0 \%$, and binding to MHC-I alleles expressed by the tumor. Selected peptides containing these neoepitopes were synthesized for functional testing. (b-c) Flow cytometry analysis of IFN $\gamma^{+}$CD8 T cells after overnight stimulation of the post-treatment patient PBMC with different peptides pools (33 peptides in 
9 pools, each peptide was present in 3 separate pools) or DMSO as control. (d) Reactivity to two peptides (p15 and p16) with the mutated (mt) or the germline (wt) KPNA2 amino acid sequence was tested in IFN $\gamma$ ELISPOT assay. Measurements (circles) including means (lines) are shown ( $\mathrm{n}=2$ independent samples). The dotted line indicates 50 spots per well (cut-off for positive signal). (e) KPNA2 mutation-reactive CD8 T cells were identified by intracellular IFN $\gamma$ expression and sorted for TCR-seq analysis. Frequency of the TCR clones from the sorted p15- and p16-reactive CD8 T cells in the patient blood at baseline (BL) and after treatment start. (f) $K P N A 2$ gene expression measured by RT-qPCR ( $\mathrm{n}=2$ for 0 and 1 X 8 Gy, and $n=4$ for 3 X 8 Gy) in a NSCLC PDX tumor 24 hours after in vivo irradiation. Expression levels were normalized 0 Gy group average. Measurements (circles) including means (lines) are shown. 